Arthroskopie $2013 \cdot 26: 89-90$

DOI 10.1007/s00142-012-0733-9

Online publiziert: 9. Mai 2013

(c) Springer-Verlag Berlin Heidelberg 2013
P.U. Brucker ${ }^{1}$ - M.A. Zumstein ${ }^{2}$

${ }^{1}$ Abteilung für Sportorthopädie am Klinikum rechts der Isar, Technische Universität München

${ }^{2}$ Schulter, Ellbogen und Sportorthopädie, Universitätsklinik für Orthopädische

Chirurgie und Traumatologie, Inselspital, Universität Bern

\title{
Intraoperative biologische Augmentation
}

Sehr geehrte Leserinnen und Leser,

Bei unserer regelmäßigen operativen Tätigkeit stehen wir häufig vor dem Problem, dass das intrinsische Heilungspotenzial verschiedener Gewebe des Stütz- und Bewegungsapparates einer relevanten Limitation unterliegt. Wir alle kennen aus eigener Erfahrung die Situation während der Operation, bei welcher gering vaskularisierte oder bereits degenerierte Gewebestrukturen nach einer Ruptur oder bei Defekten durch chirurgische Maßnahmen wieder refixiert oder rekonstruiert werden sollen. In diesen Fällen würden wir uns sicherlich eine bessere Gewebequalität oder Vaskularisation wünschen, um eine bestmögliche Heilung zu gewährleisten. Doch können wir diese limitierte Heilungskapazität durch gezielte operative Maßnahmen beeinflussen bzw. sogar verbessern?

\section{》) Können wir die limitierte Heilungskapazität operativ beeinflussen?}

Blicken wir einmal in die nähere Vergangenheit der orthopädisch-unfallchirurgischen Forschungshistorie zurück: Hier lag im letzten Jahrzehnt des 20. und zu Beginn des 21. Jahrhunderts ein wesentlicher Forschungsschwerpunkt in der Biomechanik von intakten und verletzten Strukturen des muskuloskeletalen Systems sowie der biomechanischen Belastbarkeit chirurgischer Refixations- und Rekonstruktionsmöglichkeiten zum Operationszeitpunkt. Dabei wurden zweifellos großartige Fortschritte in der Entwick- lung von Stabilisierungs-, Refixationsund Rekonstruktionstechniken unter dem Aspekt einer optimierten Primärstabilität erzielt. Allerdings traten hierbei zum Teil Probleme in der (Ein-)Heilungsphase auf, welche aufgrund der Kompromittierung der lokalen biologischen Verhältnisse per se oder durch die primärstabilen Refixations- bzw. Rekonstruktionstechniken mitverursacht wurden. Vor diesem Hintergrund sowie dem Problem der limitierten Heilungskapazität war in den letzten Jahren ein Fokuswechsel in der orthopädisch-unfallchirurgischen Forschung in Richtung biologischer Verfahren zu verzeichnen. Zukünftig könnten diese Verfahren zur optimierten Behandlung von Verletzungen und Schäden des muskuloskeletalen Systems einen relevanten Beitrag leisten.

Unter diesen sog. biologischen Verfahren können die Verwendung spezifischer Wachstumsfaktoren, zellbasierte Therapien und das weite Feld des „Tissue engineerings" subsumiert werden.

$\checkmark$ Durch einen enormen

Wissenszuwachs in der Grundlagenforschung wurde der Weg zur Herstellung und Isolation von Wachstumsfaktoren und körpereigenen Stammzellen geebnet.

So finden diese Verfahren zunehmend Einzug im klinischen bzw. operativen Alltag. Ein regelrechter Boom ist in den letzten Jahren bei der Verwendung von thrombozytenreichen Konzentraten zu verzeichnen gewesen, da diese einfach, schnell und kostengünstig als autologes Substrat aus dem Patienten direkt gewon- nen und sofort wieder nach entsprechender Präparation reappliziert werden können. Hier ist insbesondere darauf hinzuweisen, dass es neben der großen intraund interindividuellen Variabilität nicht nur das eine thrombozytenreiche Konzentrat gibt, sondern es je nach Prozessierung bzw. Präparation sehr unterschiedlich zusammengesetzt ist und entsprechend auch unterschiedliche biologische Effekte und Wirkungen zeigen kann.

Wir haben uns aufgrund der Aktualität dieser Thematik angenommen und aus Sicht des Operateurs bzw. Arthroskopeurs die erwähnten biologischen Verfahren unter dem Aspekt der intraoperativen biologischen Augmentation betrachtet. Der Fokus liegt hierbei auf den Geweben des Bewegungs- und Stützapparates, die insbesondere in der Gelenkchirurgie operativ adressiert werden. Die einzelnen Übersichtsartikel befassen sich näher mit biologischen Augmentationstechniken am Knochen, am Knorpel, an den Menisken, den Ligamenten und den angrenzenden Sehnen sowie deren Enthese.

Auch wenn oder gerade weil neuartige und zukunftsträchtige biologische Techniken vermehrt und sehr schnell den Weg in den klinischen Alltag und in den Operationssaal finden, müssen diese kritisch hinterfragt und unter wissenschaftlichen Gesichtspunkten sachlich korrekt aufgearbeitet und evaluiert werden. Diesem Aspekt trägt der letzte Übersichtsartikel Rechnung, der den Status quo der derzeitig verfügbaren und zugelassenen biologischen Augmentationstechniken hinsichtlich deren wissenschaftlicher Evidenz aufarbeitet und kritisch beleuchtet. 
Auch wenn die bisherige Datenlage bei Weitem noch nicht ausreicht, um die Evidenz von intraoperativen biologischen Augmentationstechniken abschließend beurteilen zu können, so sind wir der Überzeugung, dass hier noch großes Potenzial besteht, mit derartigen Techniken unsere operativen Ergebnisse in $\mathrm{Zu}$ kunft nicht nur relevant zu verbessern, sondern auch den postoperativen Heilungsprozess zu verkürzen bzw. weiter zu optimieren. Von daher möchten wir Ihnen nicht nur die Grundlagen von biologischen Augmentationsverfahren darlegen, sondern auch gewebespezifische Anwendungsmöglichkeiten dieser Verfahren praxisorientiert näherbringen. Es werden arthroskopische, aber auch Miniopen- bzw. offene biologische Augmentationstechniken behandelt, die Sie zum Teil bereits kennen und wahrscheinlich auch schon einsetzen. Darüber hinaus werden Sie auch neuere Techniken kennenlernen, die Sie vielleicht in Zukunft auch bei Ihren Patienten in Erwägung ziehen, mit dem Ziel, das für den Patienten bestmögliche operative Ergebnis zu erzielen.

Als Herausgeber dieses Themenheftes war uns hierbei ein wesentliches Anliegen, klinikübergreifende Übersichtsartikel zu präsentieren, um - soweit möglich eine unidirektionale expertenspezifische Philosophie zu vermeiden. So ist es gelungen, für alle Artikel jeweils mehrere Spezialisten zu gewinnen, die die jeweiligen Artikel klinikübergreifend gemeinsam verfasst haben. Dabei konnte von allen Autoren eines Beitrags ein tragbarer Konsens erarbeitet werden, was trotz moderner Kommunikationsverfahren ein beträchtlicher logistischer Aufwand war. Ihre

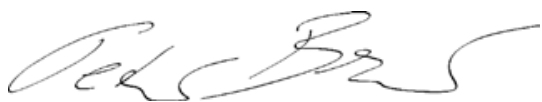

Peter Brucker

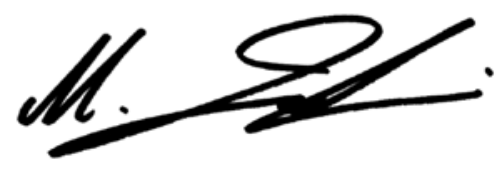

Matthias Zumstein

\section{Korrespondenzadresse}

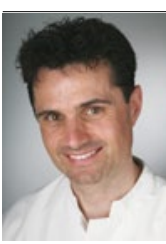

PD Dr. P.U. Brucker

Abteilung für Sportorthopädie am Klinikum rechts der Isar, Technische Universität München

Ismaninger Str. 22, 81675 München peter.brucker@ Irz.tu-muenchen.de

PD Dr. M.A.Zumstein
Schulter, Ellbogen
und Sportorthopädie,
Universitätsklinik für
Orthopädische Chirurgie und
Traumatologie, Inselspital,
Universität Bern
CH-3010 Bern
Schweiz
matthias.zumstein@insel.ch

Interessenkonflikt. Die korrespondierenden Autoren geben an, dass kein Interessenkonflikt besteht.

\section{Lumbale Dekompressionsoperationen}

Lumbale Dekompressionsoperationen sind die am häufigsten durchgeführten Wirbelsäulenoperationen. Aus klinischen Studien ist bekannt, dass dabei das klinische „Frühergebnis" in der Regel das Endergebnis determiniert. Das Frühergebnis wiederum, wird im Wesentlichen bestimmt durch die Effizienz der Dekompression und die mit der Operation einhergehenden perioperativen Morbidität.

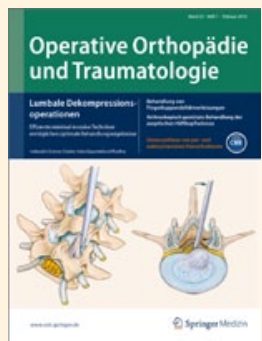

In Ausgabe 1/2013 der Zeitschrift Operative Orthopädie und Traumatologie werden 4 Operationstechniken vorgestellt die über minimalinvasive Zugänge

eine effiziente Dekompression neuraler Strukturen ermöglichen.

Beiträge des Schwerpunkthefts sind u.a.:

- The translaminar approach for cranially extruded lumbar disc herniations

- Microsurgical extraforaminal decompression of lumbar root canal stenosis

- Dekompression der lumbalen Rezessusstenose

- Selektive, mikrochirurgische „Crossover"-Dekompression mehrsegmentaler lumbaler Spinalstenosen

Bestellen Sie diese Ausgabe zum Preis von 71,- EUR zzgl. Versandkosten bei Springer Customer Service Center Kundenservice Zeitschriften Haberstr. 7

69126 Heidelberg

Tel.: +49 6221-345-4303

Fax: +49 6221-345-4229

E-Mail: leserservice@springer.com

Suchen Sie noch mehr zum Thema? Mit e.Med, dem Online-Paket von Springer Medizin, können Sie schnell und komfortabel in über 500 medizinischen Fachzeitschriften recherchieren.

Weitere Infos unter: springermedizin.de/eMed. 